MATHEMATICS OF COMPUTATION

Volume 68, Number 225, January 1999, Pages 389-393

S 0025-5718(99)00991-6

\title{
ADVANCES IN ALIQUOT SEQUENCES
}

\author{
MANUEL BENITO AND JUAN L. VARONA
}

\begin{abstract}
In this paper we describe some advances in the knowledge of the behavior of aliquot sequences starting with a number less than 10000 . For some starting values, it is shown for the first time that the sequence terminates. The current record for the maximum of a terminating sequence is located in the one starting at 4170 ; it converges to 1 after 869 iterations getting a maximum of 84 decimal digits at iteration 289.
\end{abstract}

For a positive integer $n$, let $\sigma(n)$ denote the sum of the divisors of $n$, and $s(n)=$ $\sigma(n)-n$. A perfect number is a number $n$ such that $s(n)=n$, and an amicable pair of numbers is $(n, m)$ satisfying $s(n)=m, s(m)=n$. In a similar way, cycles of numbers $\left(a_{1}, a_{2}, \ldots, a_{l}\right)$ such that $s\left(a_{i}\right)=a_{i+1}$ for $1 \leq i \leq l-1$ and $s\left(a_{l}\right)=a_{1}$ are known as aliquot cycles or sociable numbers.

An aliquot sequence is $\left\{s^{k}(n)\right\}_{k=0}^{\infty}$ defined by taking $s^{0}(n)=n$ and $s^{k+1}(n)=$ $s\left(s^{k}(n)\right)$. For one of these sequences, there are four possibilities: $(i)$ it terminates at 1 (the previous term being a prime), (ii) it reaches a perfect number, (iii) it reaches an amicable pair or a cycle, $(i v)$ it is unbounded. The Catalan-Dickson conjecture [1], [2] says that (iv) does not actually happen. But other researchers disagree with this conjecture and think that there are unbounded sequences; in fact, the alternative conjecture from Guy-Selfridge [6] (see also [3], [4], [5]) states that there are many sequences that go to infinity, perhaps almost all those that start at an even number.

The nicest way of disproving the Catalan-Dickson conjecture would be to find an $n$ so that $s^{k}(n)>s^{k-1}(n)$ for every $k$. Although it seems likely that such $n$ does not exist, H. W. Lenstra has proved that, for every $k$, there is an $m$ so that $m<s(m)<\cdots<s^{k}(m)$ (the proof is constructive and it can be found in [3]).

Several papers deal with the behavior of these sequences. The reader will find an extensive bibliography on this subject in [5]. But, before going on to explain our results, we will summarize the main computational advances in the study of aliquot sequences.

The smallest $n$ for which there was ever doubt was 138, but Lehmer showed that the sequence terminates at $s^{117}(138)=1$. Since then, the first number whose behavior is not known is 276. Many researchers have investigated the behavior of

Received by the editor October 25, 1996 and, in revised form, February 26, 1997 and July 24, 1997.

1991 Mathematics Subject Classification. Primary 11Y55; Secondary 11A25.

Key words and phrases. Aliquot sequences, sum of divisors, perfect number, amicable pair, sociable numbers, aliquot cycles.

Research of the second author supported by grant PB93-0228-C02-02 of the DGICYT.

(C)1999 American Mathematical Society 
this sequence (for instance, G. A. Paxson, H. Cohen, D. H. Lehmer, H. J. Godwin, J. L. Selfridge, M. C. Wunderlich, T. Struppeck, R. K. Guy, A. Guy and M. Dickerman), but it is not yet known if its end exists.

But there are many other sequences whose end is in doubt. For instance, there are five main sequences starting in a number smaller than 1000 whose behavior is unknown (Lehmer five: 276, 552, 564, 660, and 966). Before the year 1980, there were fourteen main sequences starting between 1000 and 2000 (Godwin fourteen), but at the present time only twelve remain unknown (Godwin showed that the 1848 sequence terminates, as did Dickerman for the 1248 sequence). In [4], a table is included showing the status of the sequences starting with numbers less than or equal to 7044. Finally, the latest results in this field can be found in Chapters B6 and B7 in [5]; here it is stated that "we have found that those for 2580, 2850, 4488, $4830,6792,7752,8862$ and 9540 also terminate".

We have been checking the aliquot sequences for numbers under 10000. We have used PARI-GP ${ }^{1}$ and, mainly, UBASIC, ${ }^{2}$ and we have run the programs on about twenty computers from the Universidad de La Rioja and the Instituto P. M. Sagasta (Logroño, Spain) for about two years, mostly during nights and weekends. The main factoring method used has been a combination of both the elliptic curve and the multiple polynomial quadratic sieve algorithms, and we have checked the primality of the factors with the APR primality test. Besides, we wanted to avoid unexpected errors so we have made a further check of the files containing the sequences. For this task, we have written a program using the MIRACL ${ }^{3}$ package. This program allows us to verify the already computed sequences easily and quickly; we have executed this program on other computers than the ones used to calculate the sequences.

For any sequence, we have stopped the calculations when both of the following happen: $(i)$ it has a driver (see [6]), which implies that it is in a stable increasing situation, without immediate hope of finding its end; (ii) it has reached a term big enough (75 digits) and whose factorization is not easily found. To increase the number of digits of a sequence may require a lot of iterates, and we are dealing with more than 80 sequences. This involves a very large amount of computer time. For instance, we spend about three quarters of an hour to factor a number with 60 decimal digits in a Pentium 100 computer with the multiple polynomial quadratic sieve algorithm; similarly, one hour and a half for one with 65 digits, 5 hours for one with 70 digits, 18 hours for one with 75 digits, one day and a half for one with 80 digits, 4 days for one with 85 digits, and two weeks for one with 90 digits. So, finally, we stopped computing on February 13, 1997.

In our study, we have achieved several advances over the results of the aforementioned authors. Some of our conclusions follow:

- We have increased the number of iterations in each sequence. In all the cases, the last term reached has at least 75 decimal digits.

- In connection to the most pursued sequence, the one beginning with 276 , we have reached 913 iterates without finishing. We have stopped at a term that has 90 decimal digits. Also, we have made a special effort with Lehmer and Godwin sequences.

\footnotetext{
${ }^{1}$ C. Batut, D. Bernardi, H. Cohen and M. Olivier, available by anonymous ftp from megrez.ceremab.u-bordeaux.fr in /pub/pari.

${ }^{2}$ Yuji Kida, available by anonymous ftp from rkmath.rikkyo.ac.jp in /ubibm.

${ }^{3}$ Michael Scott, available by anonymous ftp from ftp.compapp.dcu.ie in /pub/crypto.
} 
- The sequence starting at 8262 converges to 1 after 773 iterations, 317 being the previous prime. Its maximum is

$$
\begin{aligned}
& s^{444}(8262)=1058123957450778949935095186502241023325216959345026 \\
& =2 \cdot 223 \cdot 8831 \cdot 1256153653 \cdot 7873472183 \cdot 27163313185808805688909099,
\end{aligned}
$$

a number of 52 decimal digits.

- The sequence starting at 7080 converges to 1 after 1264 iterations, 37 being the previous prime. Its maximum is

$$
\begin{aligned}
& s^{539}(7080)=3356676040772699493933846784218306089052226182418412008219112234936 \\
& =2^{3} \cdot 419 \cdot 34110465457 \cdot 1418417145283289 \cdot 20697305061593509314923291434129565941,
\end{aligned}
$$

a number of 67 decimal digits.

- The sequence starting at 3556 converges to 1 after 2058 iterations, 59 being the previous prime. Its maximum is

$s^{551}(3556)=476372841845828677729108156155573671704082265291532247964505425538142202028$ $=2^{2} \cdot 503 \cdot 24359 \cdot 7455102267576203135197 \cdot 100819403542569743 \cdot 12931884982259497162000565321$,

a number of 75 decimal digits.

- The sequence starting at 4170 converges to 1 after 869 iterations, 79 being the previous prime. Its maximum is

$s^{289}(4170)=329561080342477212747203692863366213833838703158858822327064032192093690321488891836$ $=2^{2} \cdot 41 \cdot 97 \cdot 20374357 \cdot 1559593537 \cdot 651966073954976081342107597832287652091395156174990523498331163$,

a number of 84 decimal digits. It is a new record for the maximum of a terminating sequence (the previous record was the 1248 sequence, which terminates after reaching a maximum of 58 digits). We found it on December 22, 1996.

- Among the sequences that finish in a cycle, the one that reaches the largest maximum is the one starting at 7422 . For this sequence, we have $s^{332}(7422)=$ 2924 and $s^{333}(7422)=2620,(2620,2924)$ being an amicable pair. The sequence maximum is $s^{156}(7422)=1269528720481893811316=2^{2} \cdot 13 \cdot 109$. $131 \cdot 110323 \cdot 15497988349$. (The same happens with the 7434 and 9894 sequences, which coincide with the sequence corresponding to 7422 after a few iterations.)

Finally, in Table 1 we summarize our work with aliquot sequences starting with $n<10000$. In this table we show the main sequences whose status is yet unknown (by main we mean that all the other sequences with unknown end merge with any sequence in the table; in this case, we only show in the table the sequence that starts in the smaller number). We also include the number of decimal digits of the last term $s^{k}(n)$ reached for each sequence and the driver in that stage. This table is similar to the one in [4], but now the last term computed for every item is between $10^{20}$ and $10^{40}$ times bigger (also, the last number analyzed in [4] is 7044). It is important to note that, for every sequence in the table, we have given up computing iterations at a number containing not only a guide, but also a driver. As a consequence, no sequences are expected to start decreasing after only a few iterations.

The complete computations used to work out this table, and the last term reached for any sequence, can be obtained from the authors upon request. Also, they are available by anonymous ftp from navalsaz.unirioja.es in the directory /pub/aliquot. Equally, we have created a web page about aliquot sequences: http://www.unirioja.es/dptos/dmc/jvarona/aliquot.html. This provides a good starting point for future work. 
TABLE 1. Aliquot sequences whose end is in doubt

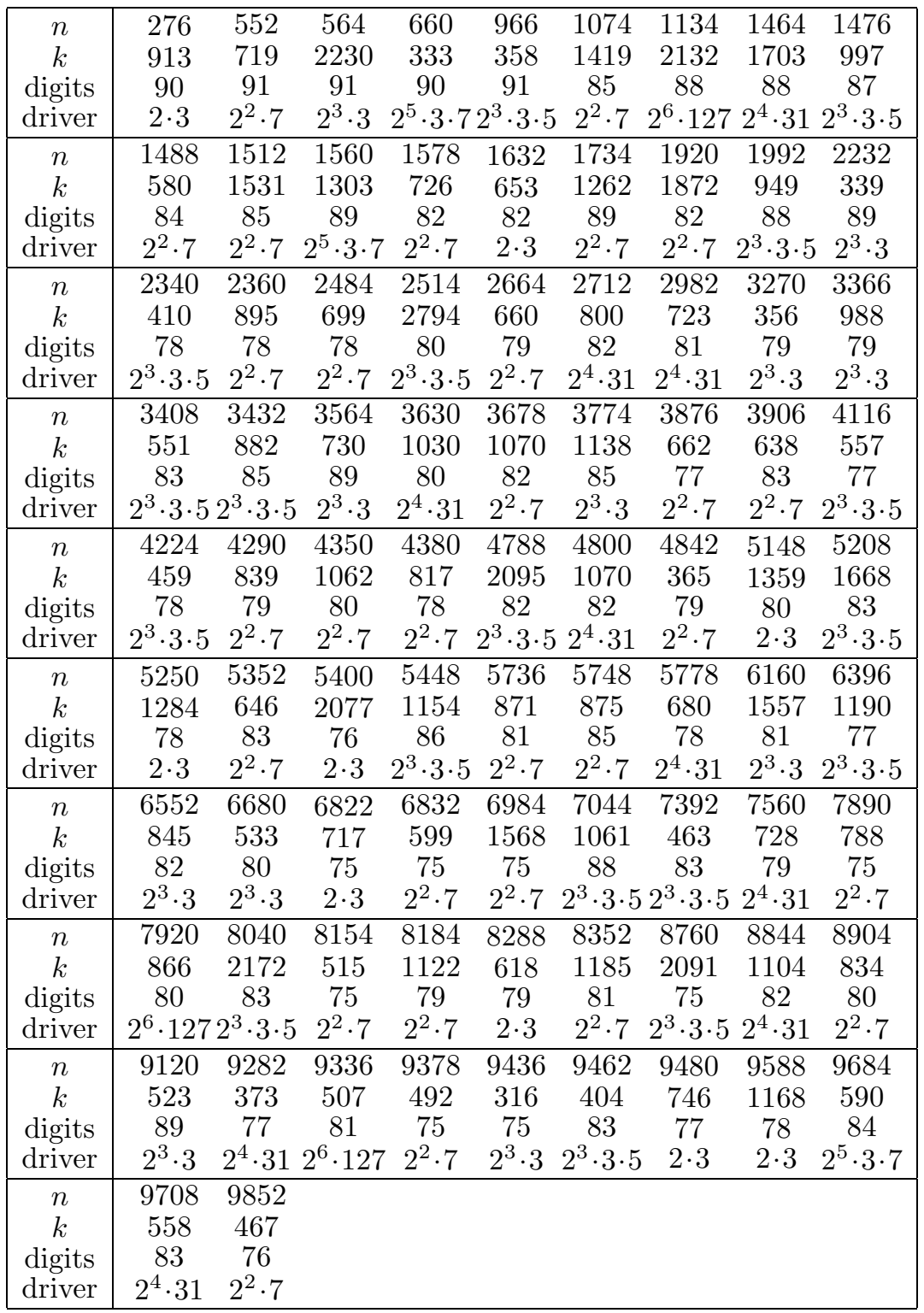

\section{ACKNOWLEDGMENT}

The authors wish to thank the referee for informing us of the work developed by W. Creyaufmueller and P. Zimmermann on this subject, that appears in the web page http://www.loria.fr/ zimmerma/records/aliquot.html. There, it can be seen that they have pursued the sequences starting from $276,552,564$, 660, 966, 1074 and 1134 up to 1059, 765, 2297, 405, 423, 1511 and 2213 iterations, respectively (updated to February 17, 1997). Also, it is announced in the web page that W. Bosma found the end of the 3556 sequence in September 1996. 


\section{REFERENCES}

1. E. Catalan, Propositions et questions diverses, Bull. Soc. Math. France 18 (1887-88), 128-129.

2. L. E. Dickson, Theorems and tables on the sum of the divisors of a number, Quart. J. Math. 44 (1913), 264-296.

3. P. Erdős, On asymptotic properties of aliquot sequences, Math. Comp. 30 (1976), 641-645. MR 53:7919

4. A. W. P. Guy and R. K. Guy, A record aliquot sequence, in Computation 1943-1993: A HalfCentury of Computational Mathematics (Vancouver, 1993), Proc. Sympos. Appl. Math. 48 (1994), Amer. Math. Soc., Providence, RI, 1994, pp. 557-559. MR 95k:11164

5. R. K. Guy, Unsolved Problems in Number Theory (2nd ed.), Springer-Verlag, 1994. MR 96e: 11002

6. R. K. Guy and J. L. Selfridge, What drives an aliquot sequence?, Math. Comp. 29 (1975), 101-107. Corrigendum, ibid. 34 (1980), 319-321. MR 52:5542; MR 81f:10008

Departamento de Matemáticas y Computación, Universidad de La Rioja, Edificio J. L. Vives, Calle Luis de Ulloa s/n, 26004 Logroño, Spain

E-mail address: mbenito@dmc.unirioja.es

Departamento de Matemáticas y Computación, Universidad de La Rioja, Edificio J. L. Vives, Calle Luis de Ulloa S/n, 26004 Logroño, Spain

E-mail address: jvarona@dmc.unirioja.es 